\section{Blut im Urin}

W. G. Guder

München, Deutschland

Synonym(e) Hämaturie; Hämoglobinurie

Englischer Begriff haematuria; haemoglobinuria, blood in urine

Definition Blut im Urin ( $\triangleright$ Hämaturie) ist eines der ältesten Krankheitszeichen. Unter diesem Begriff werden sowohl die Anwesenheit von Erythrozyten im Urin wie der Nachweis von > Hämoglobin im Urin verstanden. Je nach Menge des Bluts und der Nachweisbarkeit spricht man von $>$ Mikrohämaturie, wenn die Anwesenheit von Blut nicht mit dem bloßen Auge erkennbar ist, von \ Makrohämaturie, wenn sichtbare Rotfärbung des Urins eine Anwesenheit von Blut vermuten lässt.

Beschreibung Die Hämaturie zählt zu den häufigsten Symptomen in der ärztlichen Praxis, da sie vom Patienten selbst beobachtet wird und Anlass ist, den Arzt aufzusuchen.
Dabei ist zunächst zu unterscheiden zwischen echter Blutbeimengung in den Urin und einer Rotfärbung durch Hämoglobin, Myoglobin oder Medikamente. Zusätzlich wird der Arzt fragen oder durch eine Aufforderung zum Harnlassen ermitteln, ob die Rotfärbung in der ersten Portion, dem Mittelstrahl oder in der letzten Portion stärker ist ( $\vee$ Drei-GläserProbe). Daraus lassen sich Schlüsse über Herkunft und Mechanismus der Blutbeimengung ableiten.

Mit dem Teststreifen Blut, der auf der Reaktion des Hämoglobins als Pseudoperoxidase beruht, wird festgestellt, ob es sich um eine positive Reaktion von Hämoglobin oder Portion stärker $\triangleright$ Myoglobin handelt. Mit mikroskopischer Analyse des Sediments wird die Gegenwart von Erythrozyten, mit spezifischen immunchemischen Tests die Gegenwart von Myoglobin nachgewiesen.

\section{Literatur}

Hofmann W, Ehrich JHH, Guder WG, Keller F, Scherberich J (2011) Diagnostische Pfade bei Nierenerkrankungen. J Lab Med 35:127-146. Sowie in: Nieren- und Hochdruckkrankheiten 40:47-79 